\title{
Response Knowledge Learning of Autonomous Agent
}

\author{
Chi-kin Chow and Hung-tat Tsui \\ Visual Signal Processing and Communications Laboratory \\ Department of Electronic Engineering \\ The Chinese University of Hong Kong \\ Hong Kong SAR, China \\ E-mail: \{ckchow1,httsui\}@ee.cuhk.edu.hk
}

\begin{abstract}
In robot applications, the performance of a robot agent is measured by the quantity of award received from its response. Many literatures [1-5] define the response as either a state diagram or a neural network. Due to the absence of a desired response, neither of them is applicable to an unstructural environment. In this paper, a novel Response Knowledge Learning algorithm is proposed to handle this domain. By using a set of experiences, the algorithm can extract the contributed experiences to construct the response function. Two sets of environments are provided to illustrate the performance of the proposed algorithm. The results show that it can effectively construct the response function that receives an award which is very close to the true maximum.
\end{abstract}

\section{INTRODUCTION}

The essentiality of self-improvement $[6,7]$ in control systems becomes popular in both structural and unstructural environments, such as robotics, factory automation, and autonomous vehicles. During the processing, an agent makes a response to the environment according to its observation. The response function $R$ represents the agent's behavior and it is modeled as a function of observation from the environment: $\mathbf{P}=R(\mathbf{0})$ where $\mathbf{p}$ is the response vector and $\mathbf{0}$ is the observation vector. In the structural environment domain, state diagram is a popular representation of the response function. In this approach, each observation is defined as a state in which the weights among the states are trained by the statistical learning method such as Reinforcement Learning (RL) [1-4] and Hidden Markov Model (HMM) [5]. In this type of representation, since the observation states are finite and discrete, it is insufficient to describe the environment of real-world applications. For the continuous environment domain, regression approaches [8, 9] are used to model the agent response as a continuous function. Neural network is a common example [10]. Neural network $(\mathrm{NN})$. is able to extract patterns and detect trends that are too complex to be noticed by either humans or other computer techniques. By considering the observations $\boldsymbol{o}$ and the corresponding desired response $\mathbf{r}$ as a training set $\mathbf{S}=[\mathbf{0} \mid \mathbf{r}]$ of the $\mathrm{NN}$, the continuous response function can be computed.
On the other hand, since there is no information about the unstructural environment initially, the desired response of any observation cannot be defined. Therefore, the traditional NN approaches are not applicable. Instead of providing the desired response, the environment only returns the award of a response, i.e. award $=A(\mathbf{0}, \mathbf{r})$ where $A$ is the award function representing the characteristic of the environment. A typical example of the unknown environment application is the Prey and Predator (PP) problem, which is defined as follows:

In this model, the world consists of 2 organisms that form a prey-predator relationship. The predator aims at catching the moving prey with minimum number of steps. In addition, the predator has no knowledge on how to catch the prey initially. Therefore, it has to learn the strategy through a set of experiences which is expressed as:

- Observation $o$ is the direction from prey.

- Response $r$ is the movement direction of predator.

- Award $a$ is the change of distance between them.

Due to the absence of catching strategy, the predator will randomly generate the response to catch the prey. It is expected that some of the responses cause the predator approaching to the prey and some do not. When a sufficient number of random trials are collected, the predator can extract the strategy (response function) from the experiences.

An experience $\mathbf{E}=[\mathbf{0}, \mathbf{r} \mid$ a] of an unknown environment consists of three parts: Observation $\mathbf{0}$, Response $\mathbf{r}$ and Award a. The objective of Response Knowledge Learning (RKL) is to determine a response function that returns a response with maximum award.

$$
\text { Response Constriant : } A(\vec{o}, R(\bar{o})) \geq A(\bar{o}, \vec{r}) \text { for all } \bar{r} \quad \text { Eq. (1) }
$$

This paper is organized as follows. The objective and the proposed RKL training algorithm of are discussed in section 2. In section 3 , the architecture of a sequential training radial basis function regression network called "Memory Regression Network MRN" is introduced. Two environments are provided for response knowledge 
learning in Section 4, to illustrate the performance of the proposed algorithm. A conclusion is drawn in Section 5.

\section{RESPONSE KNOWLEDGE LEARNING}

In the robot applications, the agent keeps interact with the environment by applying a sequence of responses according to its current observation. In order to survive in the environment, the robot agent must make a response to the environment in which a maximum award is collected. The objective of RKL is to determine the response function that satisfies the response constraint described in previous experience. Different from the traditional $\mathrm{NN}$ training samples that all of them are benefit to the network; some of the RKL experiences are harmful to it. Therefore, the RKL should learn the function that receives maximum award and minimize the total punishment collected. For example, if an agent collects $n$ experiences $\mathbf{E}_{\mathbf{i}}=\left[\mathbf{0}, \mathbf{r}_{\mathbf{i}}, \mathbf{a}_{\mathbf{i}}\right]$ for $i \in[1, n]$, only the experience with maximum award is selected for learning.

Since the environment is a black-box function, we have to estimate the target environment $A_{e} \approx A$ by a regression on the collect experience set $\left\{\mathbf{E}_{i}\right\}$. Moreover, as the estimated environment function $A_{e}$ is constructed by $\left\{\mathbf{E}_{\mathbf{i}}\right\}$, the response constraint Eq. (1) can be further simplified as:

$$
A_{e}\left(\bar{o}_{i}, R\left(\bar{o}_{i}\right)\right) \geq A_{e}\left(\bar{o}_{i}, \bar{r}_{i}\right)=a_{i} \text { for all } i
$$

In the proposed algorithm, both the estimated award function $A_{e}$ and the response function $R$ are represented as the neural networks, and named as Award Network and Response Network respectively. Award Network is supported by the active experience set (AES) and represents award of the estimated environment while the Response Network returns the expected response of a given observation. Both networks are constructed by a novel sequential training radial basis function (RBF) regression network called "Memory Regression Network (MRN)". The complete algorithm is summarized below:

\section{STEP 1: AWARD FUNCTION ESTIMATION}

By given a set of distinct description about the environment (Experience Set $\left\{\mathbf{E}_{1}\right\}$ ), we firstly estimate the environment model based on the sequential training network MRN whose details are discussed at Section 3. As more experiences are collected from the environment, a more accurate award function can be estimated and hence a better response function can be established.

\section{STEP 2: MAXIMUM VIOLATED EXPERIENCE (MVE) EXTRACTION}

Given an observation $\mathbf{0}$, a well-trained response function should return the expected response $\mathbf{r}_{\mathrm{e}}$ in which the corresponding award is maximized. Therefore, an experience $\mathbf{E}_{\mathbf{i}}$ is regarded as violated from the response constraint if its expected award is smaller than the desired one, i.e. $a_{i}>A_{e}\left(\mathbf{o}_{\mathrm{l}}, \mathbf{r}_{\mathrm{e}}\right)$ where $\mathbf{r}_{\mathrm{e}}=R\left(\mathbf{0}_{\mathbf{i}}\right)$. The violated experience with maximum desired award is regarded as the MVE of current iteration.

\section{STEP 3: RETRAINING OF RESPONSE NETWORK}

After determining the current MVE, it will be appended to the active experience set $\left\{\mathbf{A E}_{\mathbf{i}}\right\}$ :

$$
\text { i.e. }\left\{\mathbf{A E}_{\mathbf{i}}\right\} \leftarrow\left\{\mathbf{A E}_{\mathbf{i}}\right\} \cup \mathbf{V E}
$$

The expanded active experience set will be used to retrain the response network using MRN.

\section{STEP 4: RESPONSE NETWORK COMPLETENESS}

The response network is well trained if there is no violated experience extracted. The flow diagram of the proposed RKL algorithm is shown in fig. 1.

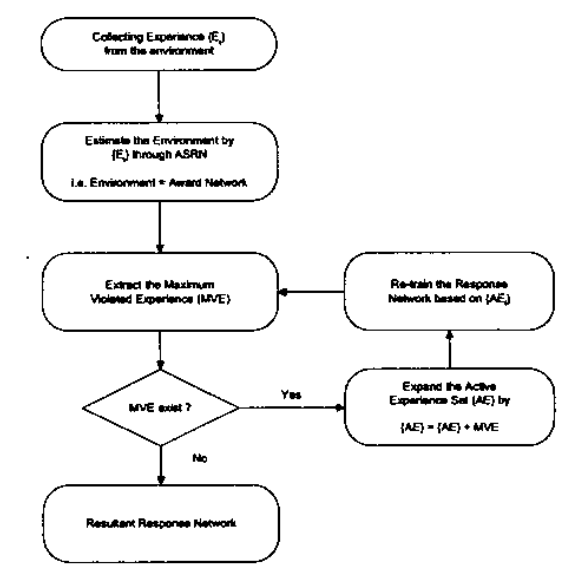

Figure 1 Flow diagram of the proposed RKL algorithm.

III. MEMORY REGRESSION NETWORK MRN

\section{A. ARCHITECTURE OF MRN}

MRN is a fixed topology RBF network. It involves two types of memory units:

1. Experience is a short-term memory unit (STMU), i.e. $\mathbf{P}$ $=[\boldsymbol{\beta}$ e $]$ where $\boldsymbol{\beta}$ and e store the input $\mathbf{x}$ and output $y$ of a training sample respectively.

2. Neuron is a long-term memory unit (LTMU), i.e. $\mathbf{H}=[\boldsymbol{\mu}$, $\sigma, M]$. Neuron is represented as a Gaussian function with the mean $\mu$, variance $\sigma$ and weight $M$.

\section{B. TRAINING ALGORITHM OF MRN}

As the present training sample $\left[x_{s} \mid y_{s}\right]$ is collected, the MRN will perform one of the 3 training phases: 
Training Procedure $=\left\{\begin{array}{cc}\text { Phase 1 } & \text { if } N_{s} \leq N_{e} \text { and } N_{s} \leq N_{n} \\ \text { Phase 2 } & \text { if } N_{s}>N_{n} \\ \text { Phase 3 } & \text { if } N_{s}>N_{e}\end{array}\right.$

where $N_{e}, N_{n}$ and $N_{s}$ are the number of experience, neuron and collected samples respectively. The details of 3 phases are described as follows:

PhaSe 1: SAMPLE MEMORIZING

The parameters of $t^{t h}$ experience $\mathbf{P}_{\mathbf{i}}=\left[\boldsymbol{\beta}_{\mathrm{i}}, \mathrm{e}_{\mathrm{i}}\right]$ are assigned as $\boldsymbol{\beta}_{\mathrm{i}} \leftarrow \mathbf{x}_{\mathrm{s}}$ and $\mathrm{e}_{\mathrm{i}} \leftarrow \mathrm{y}_{\mathrm{s}}$. In addition, the center and variance of $i^{t h}$ neuron $\mathbf{H}_{\mathrm{i}}=\left[\mu_{\mathrm{i}}, \sigma_{i}, \mathrm{M}_{\mathrm{i}}\right]$ are assigned as:

$$
\vec{\mu}_{i} \leftarrow \bar{x}_{i} \quad \sigma_{i} \leftarrow \sqrt{\frac{\min _{m \neq i}\left\|\bar{\mu}_{i}-\bar{\beta}_{m}\right\|}{-8 \ln 0.8}}
$$

Consequently, the weights of neuron set $[\mathbf{M}]$ can be determined by the Matrix Inversion method, i.e. [M] $=$ $[\mathbf{G}]^{-1}[\mathbf{Y}]$ where $G_{i, j}=\exp \left(-\left\|\mu_{\mathrm{j}}-\mu_{\mathrm{i}}\right\| / 2 \sigma_{j}^{2}\right)$ and $\mathbf{Y}=\left[\mathrm{e}_{\mathrm{i}}\right]$.

Phase 2: KNOWledge Generalization

The parameters of $i^{\text {th }}$ experience $\mathbf{P}_{\mathbf{i}}$ are assigned as $\boldsymbol{\beta}_{\mathrm{i}}$ $\leftarrow \mathbf{x}_{\mathrm{s}}$ and $\mathrm{e}_{\mathrm{i}} \leftarrow \mathrm{y}_{\mathrm{s}} \mathrm{s}$ so as to memorize the incoming sample. If there exists the neuron $\mathbf{H}_{\mathbf{k}}=\left[\boldsymbol{\mu}_{\mathbf{k}}, \sigma_{k}, \mathbf{M}_{\mathbf{k}}\right]$ such that:

1. $\left|M_{k}\right| \leq\left|M_{i}\right|$ for all $i$

2. and $\left|M_{k}\right| \leq E\left(\mathbf{x}_{\mathrm{s}}, \mathrm{y}_{\mathrm{s}}\right)$

$$
\text { where } E\left(\bar{x}_{s}, y_{s}\right)=y_{s}-\sum_{j=1}^{N_{s}} M_{j} \exp \left(-\frac{\mid \bar{\mu}_{j}-\vec{x}_{s} \|}{2 \sigma_{j}^{2}}\right)
$$

the parameters of $\mathbf{H}_{\mathbf{k}}$ will be assigned as:

$$
\bar{\mu}_{k} \leftarrow \bar{x}_{s} ; \quad \sigma_{k} \leftarrow \sqrt{\frac{\min _{m \neq k}\left\|\bar{\mu}_{k}-\bar{\beta}_{m}\right\|}{-8 \ln 0.8}} ; \quad M_{k} \leftarrow E\left(\bar{x}_{s}, y_{s}\right)
$$

Due to the neuron adjustment process listed in the above, a Gaussian regression error region is occurred. Instead of adjusting all neurons to minimize the error, a subset of them (Excited neuron set $\left\{\mathbf{O}_{\mathrm{i}}\right\}=\left\{\boldsymbol{\mu}_{0, \mathrm{i}}, \sigma_{\mathrm{o}, \mathrm{i}}, \mathrm{M}_{\mathrm{o}, \mathrm{i}}\right\}$ ) is extracted for error minimization. The procedures of extracting the excited neuron set are listed in below:

Step 1: Determine the Excitivity of $i^{t h}$ neuron $\left(C_{i}\right)$ :

$$
C_{i}=\frac{V_{i}}{U_{i}} ; \quad V_{i}=\int_{0}^{1} e^{\frac{\left|\hat{p}_{i}-\bar{x}\right|}{2 \sigma_{i}^{2}}} e^{-\frac{\left|\underline{n}_{i}-\bar{x}\right|}{2 \sigma_{r}^{2}}} d \bar{x} ; \quad U_{i}=\int_{0}^{i} e^{-\frac{\left|\beta_{i}-\bar{x}\right|}{2 \sigma_{i}^{2}}} d \bar{x}
$$

where $\mu_{e}$ and $\sigma_{e}$ is the mean and variance of $\mathbf{H}_{k}$ before the adjustment.

Step 2: Sort $\left\{C_{i}\right\}$ in descending order.

Step 3: Assume that $R(i)$ is the rank of $i^{\text {th }}$ neuron $\mathbf{H}_{\mathbf{i}}$.

Step 4: By giving the Correlation constant $\eta, \mathbf{H}_{i}$ is defined as the excited neuron if:

$$
\sum C_{h} \leq \eta \sum_{j=1}^{p} C_{j} \text { for } R(i) \geq R(h) \text { where } p \text { is the network size }
$$

In addition, the experience $\mathbf{P}_{\mathbf{k}}=\left[\boldsymbol{\beta}_{\mathrm{i}} \mathrm{e}_{\mathrm{i}}\right]$ is regarded as the element of excited experience $\left\{F_{i}\right\}=\left\{\beta_{e, i}, e_{e, i}\right\}$ if $\left\|\beta_{i}-\mu_{0, j}\right\|$ $\leq \mathrm{a} \sigma_{\mathrm{oj}}$ for some $j$. By minimizing R defined at Eq. (1), the network with minimum error can be achieved.

$$
\mathrm{R}=\sum_{i=1}^{N_{p}}\left(e_{p, i}-\sum_{j=1}^{N_{o}} M_{o, j} \exp \left(-\frac{\left\|\bar{\mu}_{o, j}-\vec{\beta}_{i}\right\|}{2 \sigma_{o, i}^{2}}\right)\right)^{2}
$$

\section{Phase 3: KNOWLedge Selection}

If there exists an experience $P_{k}=\left[\beta_{k}, e_{k}\right]$ such that

1. $E\left(\beta_{\mathrm{k}}, \mathrm{e}_{\mathrm{k}}\right) \leq E\left(\boldsymbol{\beta}_{i}, \mathrm{e}_{\mathrm{i}}\right)$ for all $i$

2. and $E\left(\boldsymbol{\beta}_{\mathrm{k}}, \mathrm{e}_{\mathrm{k}}\right) \leq E\left(\mathbf{x}_{\mathrm{s}}, y_{s}\right)$

$\boldsymbol{\beta}_{\mathbf{k}}$ and $\mathbf{e}_{\mathrm{k}}$ will be adjusted as $\mathbf{x}_{\mathbf{s}}$ and $y_{s}$ respectively.

\section{EXPERIMENTAL RESULTS}

In this section, we apply the proposed RKL algorithm to extract the responses of two environments. Both environments are the function of 1-Observation and 1Response:

$$
\begin{aligned}
& A_{1}(o, r)=\exp \left(-0.2 \times\left|\exp \left(-\frac{(o-0.5)^{2}}{0.08}\right)-r\right|-\frac{(o-0.5)^{2}}{0.08}\right) \\
& A_{2}(o, r)=\exp \left(-0.2 \times|\sin (2 \pi o)-r|-\frac{(o-0.5)^{2}}{0.08}\right)
\end{aligned}
$$

where $o, r \in[0,1]$, and the corresponding desired Response functions are:

$$
\begin{aligned}
& R_{1}(o)=\exp \left(-\frac{(o \sim 0.5)^{2}}{0.08}\right. \\
& R_{2}(o)=\sin (2 \pi o)
\end{aligned}
$$

Figure 2 shows the intensity plot of desired award functions $A_{1}$ and $A_{2}$. The x-axis represents the observation while the $y$-axis represents the response of agent to the environment. The point with higher intensity indicates its relative larger award. In these experiments, 100 experiences are provided for learning (Fig. 3). The observations/response pairs of the experiences are randomly generated within the range $G \in[0,1]$ while the corresponding awards are determined by the given award functions. Based on the experience set, the estimated award functions $A_{e, I}$ and $A_{e, 2}$ are determined and shown in fig. 4. Tables 1 and 2 list the details of the estimated award functions by the MRN. It take 1 second to construct the award networks of $A_{1}$ and $A_{2}$ with 52 and 64 neurons respectively. Figure 5 shows the resultant response network output $U_{1}$ and $U_{2}$ together with the desired response of $A_{1}$ and $A_{2}$. It takes 2 and 3 seconds to construct the response network for the award function 1 and 2 respectively. 
By given any observation $\mathbf{O}$, the response network $U(\mathbf{O})$ should return a response with maximum award. Therefore, the performance of a response function can be evaluated from its sum of award $P$ :

$$
\text { i.e. } P=\int A(\bar{o}, U(\bar{o})) d \bar{o}
$$

Tables 3 and 4 show the response network performance on both $A_{I}$ and $A_{e, I}$. It shows that the performance of response network is nearly the same of desired one. In order to illustrate the importance of RKL in robot applications, the performances of two reference response networks are studied for comparison. The first reference response network is called "Random Weight Response Network (RWRN)". The sizes of RWRNs are assigned as the same of $U_{l}$ and $U_{2}$. Figure 5 shows the examples of RWRNs. In each environment, 50 RWRNs are generated in which the average response performance is calculated. The results show that the award performances have nearly $100 \%$ improvements after the RKL process. The second reference response network is called "Max-n Response Network (Max-n)", which is constructed by training MRN with $\mathrm{n}$ highest award experiences. For each environment, we exhaustively search the value of $n$ in which the response performance is maximum. Table 3 and 4 listed the values of $n$ together with its response performances. It is shown that the performances of the best Max-n Response Networks are lower than that of the proposed one. All simulations are process at the PC platform with $1.7 \mathrm{GHz} \mathrm{CPU}$, and $256 \mathrm{MB}$ memory.

\section{CONCLUSION \& DISCUSSION}

In this article, we presented a novel RKL technique to construct a response function on unknown environment for solving an entirely new problem. The RKL consists 3 major processes: (1) Unknown Environment Modeling, (2) Active Experience Set (AES) Extraction and (3) Iterative Response Network construction. In the modeling process, the estimated award function is constructed by the collected experience set. Afterward, a set of active experiences is extracted by comparing its desired award with the one caused by the expected response. Consequently, the response network with relative

TABLE ]

Detalls of Resultant Award Network of SECTION 4

\begin{tabular}{cccc}
$\begin{array}{c}\text { Award } \\
\text { Network }\end{array}$ & $\begin{array}{c}\text { Number of } \\
\text { Experiences }\end{array}$ & $\begin{array}{c}\text { Computational } \\
\text { Time (Sec.) }\end{array}$ & $\begin{array}{c}\text { No. of non-zeros } \\
\text { magnitude agent }\end{array}$ \\
\hline $\mathrm{A}_{1}$ & 100 & 1 & 52 \\
$\mathrm{~A}_{2}$ & 100 & 1 & 64 \\
\hline
\end{tabular}

maximum reward can be achieved through training the AES with a newly introduced sequential training network MRN. In order to verify the proposed algorithm, two 1Observation 1-Response environment functions are evolved. The results show that the resultant response functions can receive a nearly maximum reward.

\section{REFERENCES}

[1] Yamaguchi, T.; Masubuchi, M.; Fujihara, K.; Yachida, M.; "Realtime reinforcement learning for a real robot in the real environment", Proceedings of the 1996 IEEE/RSJ International Conference on Intelligent Robots and Systems '96, IROS 96, Volume: 3, 4-8 Nov. 1996, Pages: 1321 - 1328.

[2] Pack Kaclbling, L:; "On reinforcement learning for robots", Proceedings of the 1996 IEEE/RSJ International Conference on Intelligent Robots and Systems '96, IROS 96, Volume: 3, 4-8 Nov. 1996, Pages: $1319-1320$.

[3] Howell, M.N.; Gordon, T.J.; "Associative reinforcement learning for discretc-time optimal control", Leaming Systems for Control (Ref. No. 2000/069), IEE Seminar, 26 May 2000, Pages:1/1 - 1/4.

[4] Kaitwanidvilai, S.; Pamichkun, M.; "Active Bayesian feature weighting in reinforcement learning robot", 2002 IEEE International Conference on Industrial Technology, 2002, lEEE ICIT '02, Volume: 2,11-14 Dec. 2002, Pages: 1090- 1095.

[5] Ogawara, K.; Takamatsu, J.; Kimura, H.; Ikeuchi, K.; "Modeling manipulation interactions by hidden Markov model", IEEE/RSJ International Conference on Intelligent Robots and System, 2002, Volume: 2, 30 Sept.-5 Oct. 2002, Pages: 1096 - 1101.

[6] Bentivegna, D.C.; Atkeson, C.G.; "Leaming from observation using primitives", Proceedings of the IEEE Intemational Conference on Robotics and Automation 2001, ICRÁ 2001, Volume: 2, 2001, Pages: $1988-1993$.

[7] Enokida, S.; Ohashi, T.; Yoshida, T.; Ejima, T.; "Stochastic ficld model for autonomous robot learning", Proceedings of the IEEE International Conference on Systems, Man, and Cybernetics 1999, IEEE SMC '99, Volume: 2, 12-15 Oct. 1999, Pages: 752 - 757.

[8] Schaal, S.; Atkeson, C.G.; "Robot leaming by nonparametric regression", Proceedings of the IEEE/RSJ/GI International Conference on Intelligent Robots and Systems '94, Advanced Robotic Systems and the Real World', IROS '94, Volume: 1, 12-16 Sept. 1994, Pages: $478-485$.

[9] Atkeson, C.G.; "Using locally wcighted regression for robot leaming", Proceedings of the IEEE International Conference on Robotics and Automation 1991, ICRA 1991,9-11 April 1991 Pages: 958 - 963 .

[10] Gross, H.-M.; Stcphan, V.; Krabbes, M.; "A neural field approach to topological reinforcement lcaming in continuous action spaces", IEEE World Congress on Computational Intelligence. The 1998 IEEE International Joint Conference on Neural Networks Proccedings, 1998, Volume: 3, 4-9 May 1998, Pages: $1992-1997$.

TABLE 2

Detalls of Resultant Response Network of SeCtSON 4

\begin{tabular}{ccc}
\hline $\begin{array}{c}\text { Response } \\
\text { Network }\end{array}$ & $\begin{array}{c}\text { Computational } \\
\text { Time (Sec.) }\end{array}$ & $\begin{array}{c}\text { No. of non-zeros } \\
\text { magnitude agent }\end{array}$ \\
\hline $\mathrm{U}_{1}$ & 2 & 10 \\
$\mathrm{U}_{2}$ & 3 & 18 \\
\hline
\end{tabular}


TABLE 3

COMPARISON Of SUMOF AWARDat SECTION4 AWARD Function 1

\begin{tabular}{ccc}
\hline Response Network & Desired Award function & Estimated Award function \\
\hline Random Weight & 0.645333 (Average) & $0.574367($ Average $)$ \\
Max-n & $0.76541(\mathrm{n}=12)$ & $0.70384(\mathrm{n}=16)$ \\
The Proposed Algorithm & 0.928238 & 0.802978 \\
Desired & 1.000000 & 0.824573 \\
\hline
\end{tabular}

TABLE 4

Comparison of Stumof awardat Section 4 award Function 2

\begin{tabular}{ccc}
\hline Response Network & Desired Award function & Estimated Award function \\
\hline Random Weight & 0.446420 (Average) & $0.408869($ Average $)$ \\
Max-n & $0.62385(\mathrm{n}=23)$ & $0.57625(\mathrm{n}=32)$ \\
The Proposed Algorithm & 0.890513 & 0.743906 \\
Desired & 1.000000 & 0.792052 \\
\hline
\end{tabular}
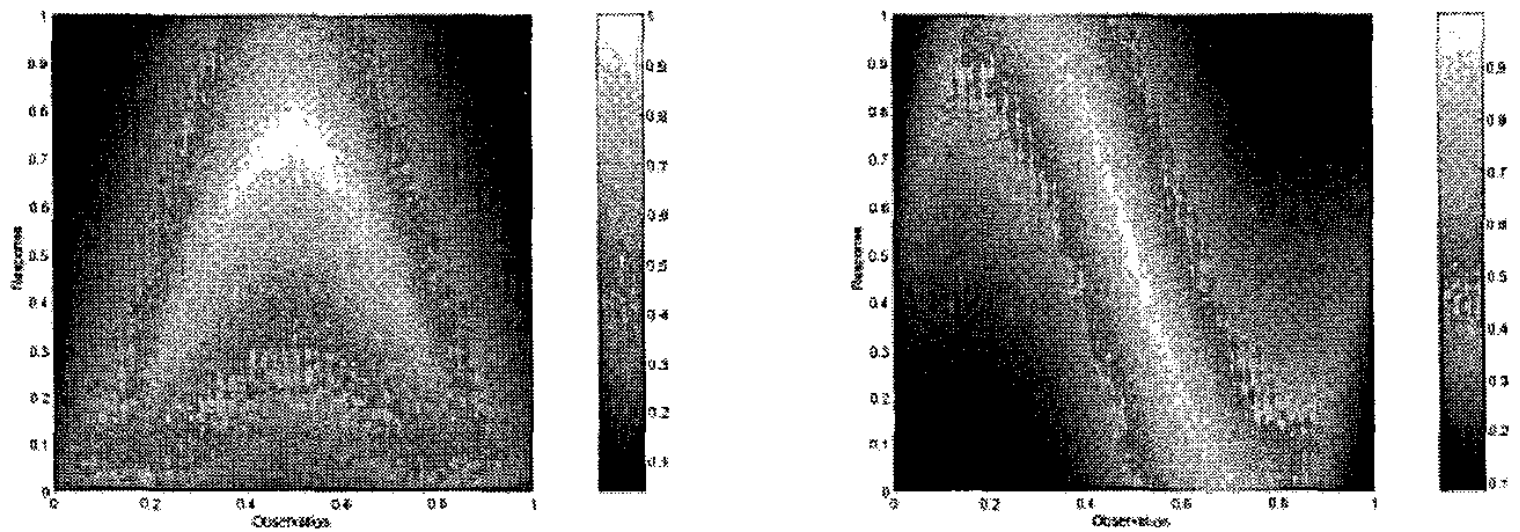

Figure 2 The desired Award Surfaces of $A_{1}$ and $A_{2}$ at Section 4
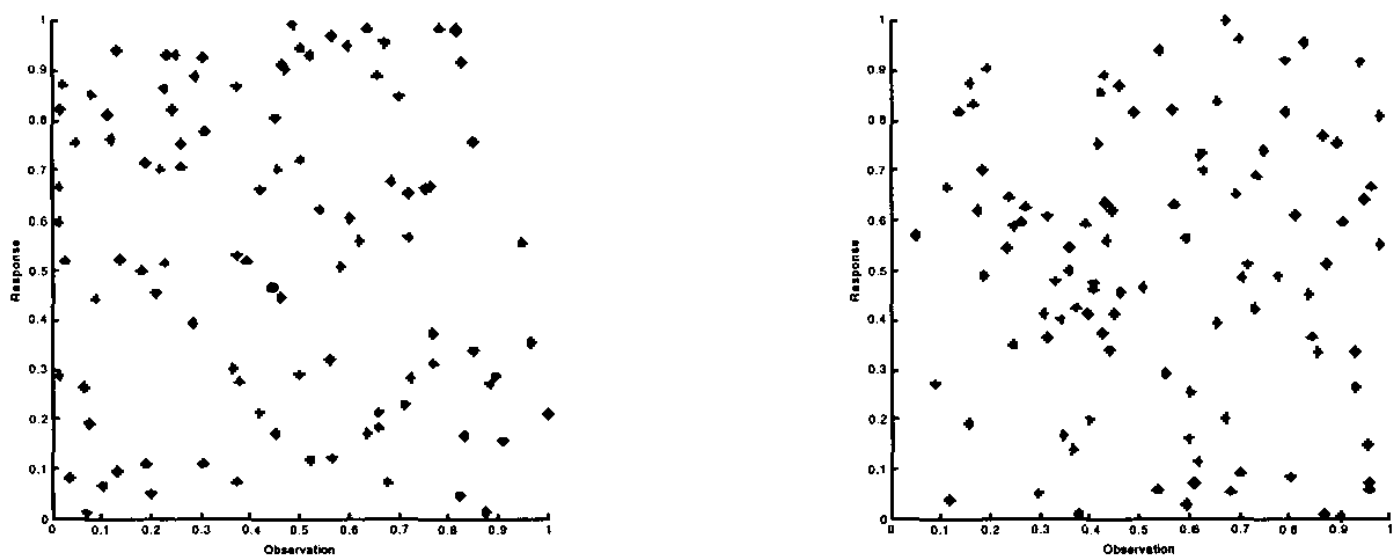

Figure 3 Distribution of experience sets of $A_{1}$ and $A_{2}$ at Section 4 

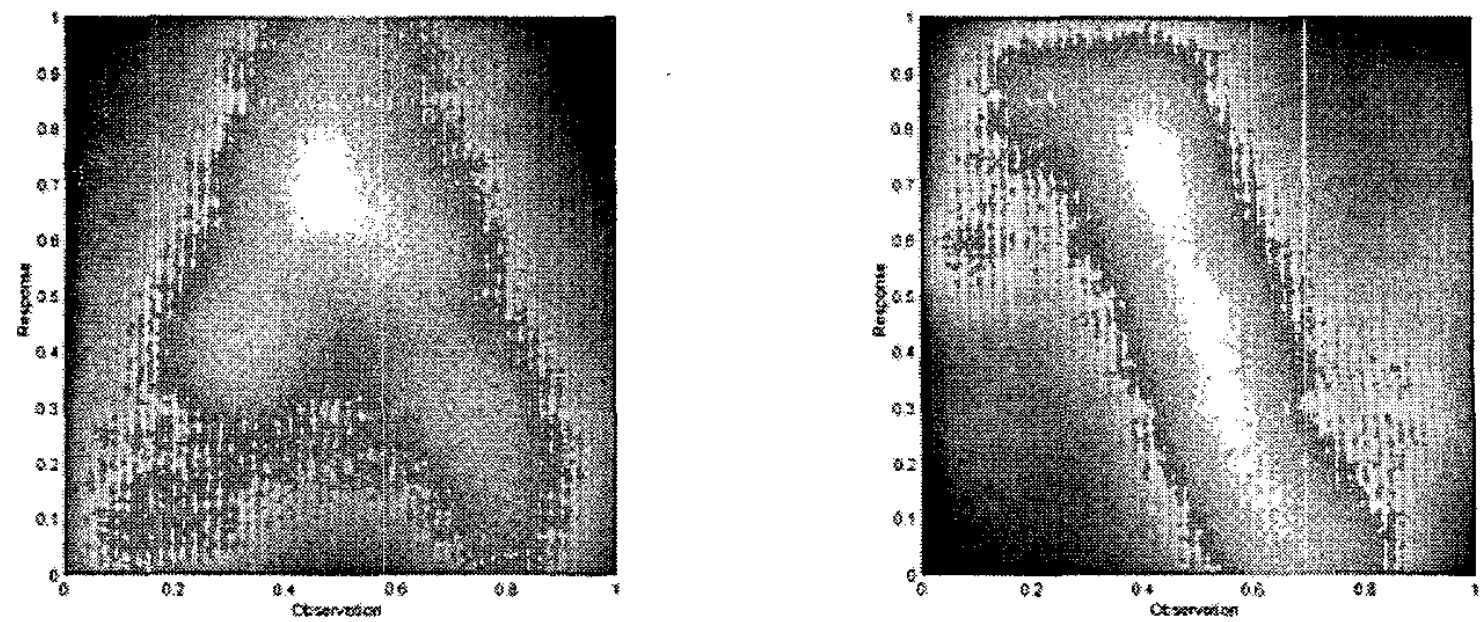

Figure 4 The estimated award surfaces of $A_{1}$ and $A_{2}$ at Section 4
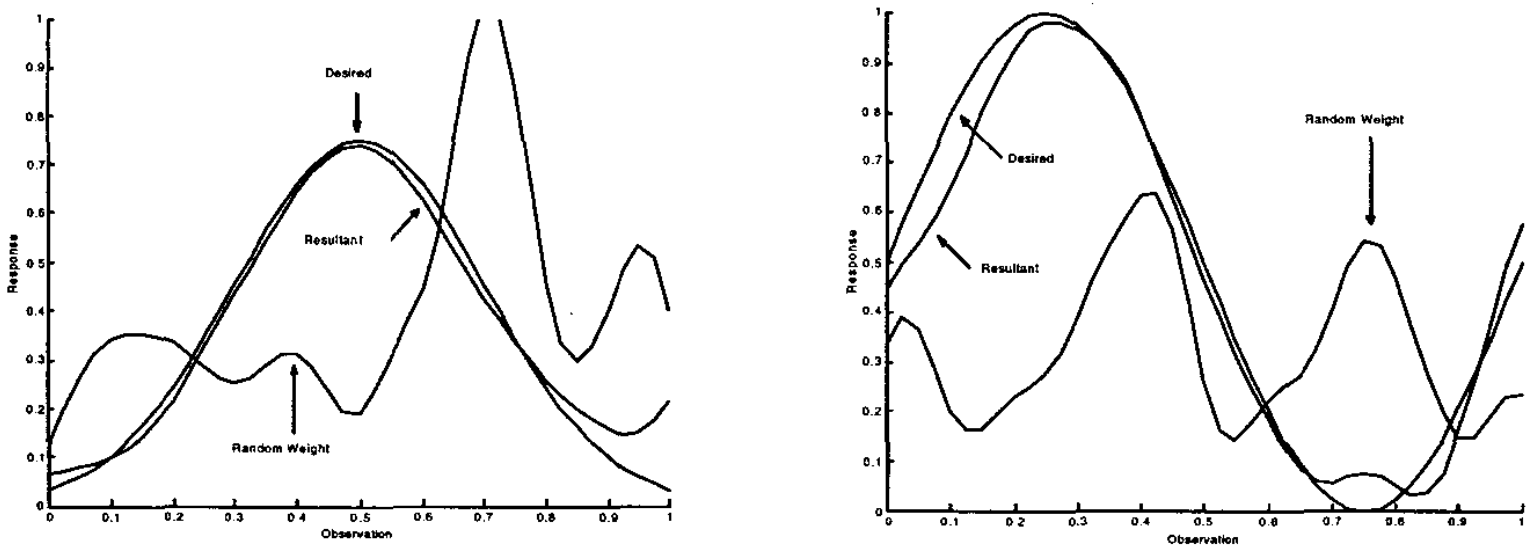

Figure 5 Comparison among the Desired, Radon Weight and Resultant Response network outputs of $A_{1}$ and $A_{2}$ at Section 4 